the oral penicillin challenge test can be safely performed in the community clinic setting.

Methods In this retrospective study the electronic medical files of 402 study participants, of ages $0-17$, were reviewed. The included patients were those flagged as allergic to penicillin, referred to a community allergy clinic between 2011-2018, were evaluated by an allergist, and underwent a penicillin or amoxicillin challenge test. The challenge test results were collected and reviewed for documentation of allergic reactions. None of the children had undergone an allergy skin or blood test prior to the challenge test. In addition, the medical files were reviewed for documentation of later use of penicillin derivatives after the challenge test.

Results Apart from a single vomiting incident during the challenge test, none of the challenge tests elicited any responses. Eighty-two (26\%) children who underwent the challenge test, used penicillin again at a later stage. Repeat diagnosis of penicillin sensitivity was recorded for 7 (3\%) children, following onset of rash following exposure to penicillin after the challenge test.

Conclusions The vast majority of penicillin allergy diagnoses among children are inaccurate, as the rash appearing during use of the medicine is unrelated to an acute allergic reaction. Viral diseases can be a significant factor underlying these rashes. The oral penicillin derivative challenge test performed in community clinics are safe, and should therefore be preferred for any child presenting late-onset rash during penicillin treatment. Widespread use of this challenge test will assist in reducing extraneous use of broad-spectrum antibiotics and resistance to these drugs.

\section{P10 MANAGEMENT OF SEVERE ATOPIC AND VERNAL KERATOCONJUNCTIVITIS}

${ }^{1}$ Marta Chlubek*, ${ }^{1}$ Pasan Fernando, ${ }^{2}$ Melanie Hingorani, ${ }^{2}$ Stephen Tuft, ${ }^{2}$ Annegret Dahlmann-Noor. 'University of Cambridge, Cambridge, UK; ${ }^{2}$ Moorfields Eye Hospital, London, UK

\subsection{6/archdischild-2019-epa.366}

Background Vernal keratoconjunctivitis (VKC) and atopic keratoconjunctivitis (AKC) are severe allergic diseases of the ocular surface which can have sight-threatening complications such as shield-shaped ulcers and plaques.

Paediatricians, general practitioners and optometrists play an important role in the early detection of severe VKC and AKC to ensure swift onwards referral to an eye clinic for treatment with topical or oral immunosuppressants or modulators and, if required, surgical intervention.

Purpose To evaluate the indications for oral corticosteroid treatment, as an indicator of severe inflammation requiring systemic immunosuppression, in the management of VKC/AKC in children, and to describe treatment outcomes.

Methods We reviewed the medical records of children up to the age of 16 years who had been prescribed oral corticosteroids for $\mathrm{VKC} / \mathrm{AKC}$ at a tertiary referral centre in the UK between 2008 and 2018. We noted age, gender, severity of corneal epithelial disease (Cameron and Moorfields grading systems), proportion of ulcers healed within 2 weeks of starting oral corticosteroids, proportion of children requiring surgical intervention, and time from starting steroids to epithelialisation. In children who received oral steroids on more than one occasion, we included the first episode only.
Results We identified 16 children and young people (median age 11.5 years, interquartile range 9-14); 15 (94\%) were boys.

Seven (44\%) children with severity grade 1 Cameron/2 Moorfields had received oral corticosteroids in addition to topical eye drops; re-epithelialisation in under 14 days was achieved in $86 \%(6 / 7)$, while $14 \%(1 / 7)$ required additional superficial keratectomy. Seven (44\%) patients with severity grade 3 (established plaque) received oral steroids; in four it was supplementary to superficial keratectomy, given for antiinflammatory purposes, and to prevent recurrence of the ulcer due to uncontrolled inflammation. In three, elective keratectomy was not carried out initially; one plaque ulcer resolved spontaneously, while two did not re-epithelialise and required surgery 48 and 63 days after oral steroids were started. Two (13\%) patients with low-grade lesions received oral therapy due to imminent risk of progression. Re-epithelialisation time was measured for one of these patients and found to be under 14 days.

Conclusions The clinical signs of severe VKC/AKC which require topical and/or systemic corticosteroids can be detected without specialist equipment, by instillation of fluorescein eye drops. Swift referral to an eye clinic allows timely treatment and optimises outcomes. We propose a simple algorithm to guide management.

\section{P11 SECONDARY LACTOSE INTOLERANCE AND COW'S MILK PROTEIN ALLERGY IN INFANTS}

${ }^{1}$ valeria novikova*, ${ }^{1}$ Natalya Bogdanova, ${ }^{1}$ Natalya Prokopyeva, ${ }^{2}$ Sergey Lapin, ${ }^{2}$ Daria Kuznetsova, ${ }^{3}$ Pavel Vorontsov. ${ }^{1}$ St. Petersburg State Pediatric Medical University, St. Petersburg, Russian Federation; ${ }^{2}$ St. Petersburg First Medical University, St. Petersburg, Russian Federation; ${ }^{3}$ medical social institution, St. Petersburg, Russian Federation

\subsection{6/archdischild-2019-epa.367}

Introduction Cow's milk protein allergy (CMPA) is one of the causes of secondary lactose intolerance (SLI) in infants.

The aim To determine the possibility of faecal tests: calprotectin, ECP and hidden blood in the stool for the diagnosis of gastrointestinal manifestations of CMPA in infants with SLI.

Patients and methods 30 children, aged from 1 to 5 months, full-term born, suffering from infantile colic and gastrointestinal disorders due to lactose intolerance, confirmed by FOG test the background of cow's milk protein intolerance, were observed. $63.33 \%$ of children were breastfed, $36.67 \%$ were mixed, received medical infant formula.CMPA in examined participants was diagnosed based on recommendations of EAACI (2014) and CoMiSS scale. SLI was confirmed by FOG, which was carried out by spectrophotometric measurement of the concentration of $\mathrm{Na}+$ and $\mathrm{K}+$ ions. Calprotectin and ECP in the stool were determined by ELISA. To detectthe hidden blood in the stool,out-of-point «Colon View» test was used. The control group consisted of 10 healthy similar aged, breastfed infants. Statistical analysis was carry out using Stat Soft Statistica 12.0. for Windows-10.

Results Participating children suffered from colic in $86.67 \%$, regurgitation in $56.67 \%$, diarrhea in $83.33 \%$, ishesion in $46.67 \%$, constipation in $13.33 \%$, blood streaks in thestool in $13.33 \%$, atopic dermatitisin $73.33 \%$. All had a combination of two or more gastrointestinal disorders.

The average level of FOG in CMPA was higher than in healthy ones $(204.94 \pm 23.56 \mathrm{Osmol} / \mathrm{kg}$. And $116.52 \pm 65.8$ Osmol/kg; P <0.001). Average values of calprotectin (463.89 
$\pm 281.4 \mu \mathrm{g} / \mathrm{g}$ and $204.45 \pm 190.29 \mu \mathrm{g} / \mathrm{g} ; \mathrm{p}>0.05)$ and ECP $(2053.13 \pm 1236.61 \mathrm{ng} / \mathrm{ml}$ and $1258.88 \pm 1012,80 \mathrm{ng} / \mathrm{ml})$ didn't differ in both groups, however, the frequency of elevated ECP levels was higher in the group of CMPA $(63 \%$ and $30 \%, \mathrm{p}<0.05)$. And the blood in the stool was detected more often $(22.2 \%$ and $0 \%, \mathrm{p}<0.05)$. The correlation between the levels of calprotectin and ECP $(r=0.55, p$ $<0.05$ ) and between the levels of FOG and ECP $(r=0.37, p$ $<0.05$ ) was found.

Conclusion Clinical diagnosis of CMPA in infants should be supplemented with coprological tests of FOG, ECP and hidden blood in the stool for the well-timed diagnosis of CMPA gastrointestinal manifestations and secondary SLI.

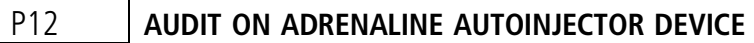 COMPLIANCE IN A PAEDIATRIC ALLERGY OUTPATIENT SERVICE}

Gearoid McGauran*, Elizabeth Murphy, Nadeem Gadelsayed, Aideen Byrne, David Coghlan. Tallaght University Hospital, Dublin, Ireland

10.1136/archdischild-2019-epa.368

Introduction Food allergy has become an increasingly common condition in the paediatric community over recent decades ${ }^{1}$. Many of these patients are at risk of developing anaphylaxis following exposure to food allergens. Adrenaline Auto-Injector (AAI) devices are prescribed to these patients to be used in the event of anaphylaxis following accidental exposure.

Guidance The Irish Food Allergy Network (IFAN) recommends that patients who are prescribed AAIs should carry two AAIs at all times. For school-going children, it is recommended that two AAIs should be stored in their school in the event of accidental exposure ${ }^{2}$.

Aims To evaluate parental compliance with carriage of AAI devices according to guidelines.

To review parental confidence on when and how to use AAIs.

Methodology A one-page questionnaire was compiled, which was approved by Tallaght University Hospital Clinical Audit Committee.

We invited parents of children attending the paediatric allergy outpatient and day-ward services who had been prescribed AAI devices to complete this questionnaire anonymously and return it to clinical staff.

This audit was carried out from October 2018 to January 2019 in five outpatient and four day-ward clinics each month. Data was compiled in a spreadsheet and interpreted.

Results 53 parents completed our questionnaire. Patients ranged from nine months to sixteen years of age.

$71 \%(n=38)$ reported carrying AAI devices. Of these, $66 \%$ $(\mathrm{n}=35)$ carried two devices.

$88 \%(n=47)$ reported that their AAIs were within date.

Of those without devices, $15 \%(n=8)$ reported AAIs were left in the car while another $11 \%(n=6)$ stated their AAIs were left at home.

Regarding confidence in AAI training, 75\% $(n=40)$ of parents surveyed felt confident in knowing when an AAI should be given, giving a score of 4 or 5 on a scale of 1 to 5. $86 \%(n=46)$ were confident in knowing how to administer an AAI device.

Conclusion Our audit shows that 1 in 3 patients who were prescribed AAIs did not carry the recommended two devices with them when attending our outpatient services. Only $75 \%$ of patients and parents felt confident in knowing when they should give an AAI. This audit highlights the importance of taking the time to educate and re-educate patients and parents. Emphasis should be made on the importance of carrying AAIs at all times.

\section{REFERENCES}

1. Ben-Shoshan M et al, Curr Allergy Asthma Rep (2012)12:346. https://doi.org/ 10.1007/s11882-012-0274-3

2. IFAN[Internet], Food Allergy in School. Cork Irl. 2017(cited Jan 2019) http://ifan. ie/childcare-schools/Save

\section{P13 IS THERE ANY RELATIONSHIP BETWEEN DEGREE OF CONTROL AND THE RISK OF EXACERBATION IN ALLERGIC ASTHMATIC CHILDREN WITH MONOSENSITISATION AND POLYSENSITISATION?}

${ }^{1}$ Ana-Maria Moiceanu Sovarel, 1,2Eugenia Buzoianu, ${ }^{3,1}$ Vlad Plesca, ${ }^{2}$ Mariana Moiceanu, ${ }^{1}$ Doina-Anca Plesca*. 'university Of Medicine And Pharmacy 'Carol Davila', Bucharest, Romania; ${ }^{2}$ Children's Clinical Hospital 'Victor Gomoiu', Bucharest, Romania; ${ }^{3}$ National Institute Of Phono-Audiology And Functional Otolaryngology 'Hociota', Bucharest, Romania

\subsection{6/archdischild-2019-epa.369}

Introduction Allergic asthma is a chronic inflammatory disease of the conducting airways that affected 1 in 10 children. Most children have allergic asthma that coincides with allergic sensitization defined by the presence of serum immunoglobulin E (IgE) antibodies and/or a positive skin-prick test to the common inhaled allergens. It is important to identify the asthma trigger factors that could worsen asthma acutely to cause an exacerbation or perpetuate chronic symptoms and airflow limitation.

Aim To assess the correlation between children with monosensitisation and polysansitisation with the asthma control status in children with allergic asthma.

To asses if the monosensitisation and polysensitisation influence the episodes of exacerbations in children with allergic asthma

Method A prospective study included 100 children aged between 5 to 18 years diagnosed with asthma and aeroallergens sensitization was conducted in 'Victor Gomoiu' Clinical Children's Hospital from January 2016 to September 2018. In each patient was assess asthma control status after 3 months. Within one year the exacerbations were registered also.

By specific serum IgE dosing, children were enrolled as mono or polysensibilized. The correlation between mono and polysensitisation and the control asthma status in children with allergic asthma was assessed using Pearson Chi Square test.

The correlation between mono and polysensitisation and the influence on the exacerbations in children with allergic asthma was assessed using Pearson Chi Square test.

Results Group 1 was formed with patients with controlled asthma and group 2 with children partially controlled and uncontrolled asthma.

39 patients were with monosensitisation; out of these 12 had controlled symptoms and 27 with uncontrolled symptoms.

61 patients were with polysensitisation; out of these 11 had controlled symptoms and 50 with uncontrolled symptoms.

The exacerbations were divided in $<2$ and $\geq 2$ in 1 year.

$<2$ exacerbation: 4 were monosensitised and 7 were polysensitised.

$\geq 2$ exacerbations: 35 patients were monosensitised and 54 were polysensitised. 